\title{
Determination of Bi Impurity in Lead Stock Standard Solutions by Hydride-generation Inductively Coupled Plasma Mass Spectrometry
}

\author{
Chang J. Park \\ Korea Research Institute of Standards and Science, P.O. Box 102. Yusong. Daejeon 305-600. Korea \\ Received October 10.2003
}

\begin{abstract}
Total impurity analy'sis of a primary standard solution is one of the essential procedures to determine an accurate concentration of the standard solution by the gravimetry. Bi impurity is determined in $\mathrm{Pb}$ standard solutions by inductively coupled plasma mass spectrometry (ICP-MS). The direct nebulization of the $\mathrm{Pb}$ standard solution produces a significant amount of the $\mathrm{Pb}$ matrix-induced molecular ions which give rise to a serious spectral interference to the Bi determination. In order to avoid the spectral interference from the interferent ${ }^{2108} \mathrm{PbH}^{-}$, the hydride generation method is employed for the matrix separation. The Bi hydride vapor is generated by reaction of the sample solution with $1 \%$ sodium borohydride solution. The vapor is then directed by argon carrier gas into the ICP after separation from the mixture solution in a liquid-gas separator made of a polytetrafluoroethylene membrane tube. The presence of $1000 \mu \mathrm{g} / \mathrm{mL} \mathrm{Pb}$ matrix caused reduction of the bismuthine generation efficiency by about $40 \%$. The standard addition method is used to overcome the chemical interference from the $\mathrm{Pb}$ matrix. Optimum conditions are investigated for the hydride-generation ICPMS. The detection limit of this method is $0.5 \mathrm{pg} / \mathrm{mL}$ for the sample solutions containing $1000 \mu \mathrm{g} / \mathrm{mL} \mathrm{Pb}$ matrix.
\end{abstract}

Key Words : Bismuth impurity, $\mathrm{Pb}$ matrix. Hydride generation, Standard addition, ICP-MS

\section{Introduction}

Single-element standard solutions are used as stock solutions for preparing calibration solutions in elemental analysis by atomic spectrometry. Accuracy of the elemental analysis relies on accuracy of the standard solution used for the calibration. Total impurity analysis of a primary standard solution is one of the essential procedures to determine the concentration of the standard solution by the gravimetry.. In this work. impurity of $\mathrm{Bi}$ is determined in a primary $\mathrm{Pb}$ standard solution prepared in this laboratory and in a commercial $\mathrm{Pb}$ stock standard solution by inductively' coupled plasma mass spectrometry (ICP-MS).

ICP-MS has attracted widespread interest because of its analytical figures of merit such as the excellent power of detection and the ability to measure isotope ratios. The most common sample introduction method for ICP-MS is the pneumatic nebulization of solutions. ${ }^{2}$ The pneumatic nebulization technique is simple to use and relatively inexpensive. but it has several disadvantages when the sample solutions contain high level of dissolved solids exceeding $1000 \mu \mathrm{g} /$ $\mathrm{mL}$. Firstly direct nebulization of the solutions can contaminate sample introduction devices. sampler/skimmer cones. ion lenses and mass filter. "The contamination causes a serious memory effect when the dissolved matrix elements have to be determined in next analyses. ${ }^{+}$The dissolved solids can also cause the gradual loss of sensitivity or the signal drift during analysis due to the decreasing ion transmission through the cones and ion lenses. ${ }^{5}$ Secondly the direct nebulization of the solutions may give inaccurate results due to the matrix effect which is associated with the physical processes governing the transfer of solutions to the nebulizer. the formation of aerosols. transport of the aerosols into the plasma and the transmission of ions through the interface and the ion lenses. ${ }^{6}$ The matrix effect results in differences between the instrument responses for the sample and the calibration standard solutions. Thirdly the direct nebulization of the solutions may generate the matrix-induced molecular ions that interfere with the analyte ions. ${ }^{7}$ In order to alleviate these drawbacks. the stock standard solutions were diluted to $10 \mu \mathrm{g} / \mathrm{mL}$ and then nebulized into the plasma for the impurity analysis. However. 100 fold dilution of the stock standard solution was not enough to prohibit the formation of the interfering matrix-induced molecular ion. ${ }^{12} \mathrm{PbH}^{-}$. Hence it was necessary to separate $\mathrm{Bi}$ analyte from the $\mathrm{Pb}$ matrix somehow.

$\mathrm{Bi}$ was determined in steels ${ }^{\S}$ and alloys $\mathrm{s}^{9}$ because its addition to the metallurgical materials can affect their property either positively or negatively. $\mathrm{Bi}$ was also determined in geological materials because it can be used in the mineral exploration as a pathfinder. ${ }^{1 / 1}$ In the previous works of the $\mathrm{Bi}$ determination, regardless of the detection methods. the hydride generation method was exclusively employed for the sample pretreatment. This paper describes a continuousflow hydride generation with a liquid-gas separator made of a Teflon membrane tube that efficiently generates and transports $\mathrm{BiH}_{3}$ into the plasma. Sample introduction with the hydride generation was used for the separation of the $\mathrm{Pb}$ matrix to eliminate the spectral interference from the matrixinduced molecular ion. The $\mathrm{Pb}$ matrix in the sample solutions. however. lowered the Bi hydride generation efficiency to cause the chemical interference in the determination of $\mathrm{Bi}$. The standard addition method was used for the quantification to overcome the chemical interference effect. 


\section{Experimental Section}

Instrumentation. All measurements were carried out on an ГL.Г:MГNT high-resolution ICP-MS instrument (Finnigan MAT, Bremen, Germany). The instrument provides three fixed resolution settings $(m / \Delta m=300,3000$ and 8000$)$. All data were acquired in low-resolution mode $(m / \Delta m=300)$. A continuous-flow hydride generation system is described in Figure 1. The sample and sodium borohydride solutions are mixed in a Teflon tube (i.d. $=0.07 \mathrm{~cm}$ and length $=50 \mathrm{~cm}$ ). The hydride vapor with the evolved hydrogen was separated from the mixed solution in a liquid-gas separator made of a Teflon membrane tube. Through the large surface of the Teflon membrane tube the gaseous hydride diffused into the glass chamber, which alleviated the pulsation effect commonly observed in the conventional $U$ tube licjuid-gas separator. The pulsation effect could give such adverse effects as the plasma flickering, and generation and injection of aerosols of the mixture solutions into the plasma. "An exit end of the licuid-gas separator was a socket joint and was directly connected to the torch. The hydride vapor was swept into the plasma by the argon carrier gas. Details of the instrument component, typical operating conditions and data acquisition parameters are given in Table 1.

Reagents and Materials. The primary $\mathrm{Pb}$ standard solution (1000 $\mu \mathrm{g} / \mathrm{ml}$ ) for the impurity analysis was prepared

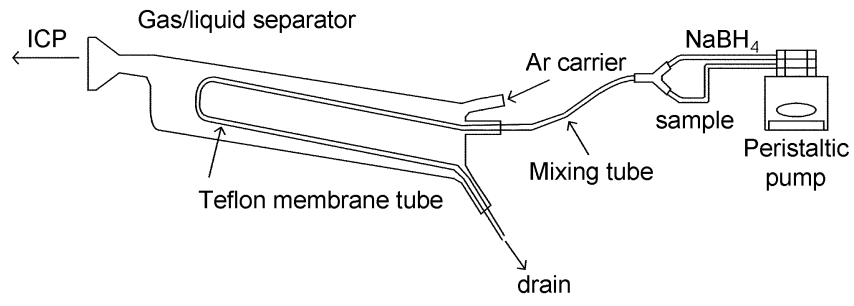

Figure 1. Schematic diagram of apparatus for Bi hydride generation.

Table I. Oparating Conditions and Lata Acgujsition Parameters of High-Resolution ICP-MS

\begin{tabular}{|c|c|}
\hline$\overline{K C P}$ & \\
\hline Rf power. W & 1300 \\
\hline Sample injection rate. $\mathrm{mL} / \mathrm{min}$ & 1.0 \\
\hline Val3l ${ }_{4}$ injection rale. $\mathrm{ml} / \mathrm{m}$ in & 1.0 \\
\hline Argon gas flow rates. 1 min & \\
\hline Coolant & 13.4 \\
\hline Auxiliary & 0.7 \\
\hline Carricr & 0.56 \\
\hline Samplar cont & Copper. 1.0 mm oritice diameter \\
\hline Skimmer cone & Copper. $0.7 \mathrm{~mm}$ orifice diameter \\
\hline Data acquisition & \\
\hline No. of passes & 3000 \\
\hline Mass window(\%) & 100 \\
\hline Search window (\%) & 100 \\
\hline Inteeration window $(\%)$ & 50 \\
\hline Simples per peak & 20 \\
\hline Sample times s: & 0.002 \\
\hline Seuling limes s & 0.001 \\
\hline
\end{tabular}

by dissolution of pure lead metal shots $(99.9995 \%$, Aldrich. Milwaukee, W1, USA). A stock standard solution (1000 mg/ $\mathrm{kg}$ ) of bismuth was purchased from National Institute of Standards and Technology (NIST, Gaithersberg, MD, USA). Working standard solutions were made by serial dilution of the stock standard solution. Sodium borohydride solution $(1 \% \mathrm{~m} / \mathrm{v})$ was daily prepared by dissolving $1 \mathrm{~g}$ of sodium borohydride powder (99\%, Aldrich, Milwaukee, WI, USA) in $100 \mathrm{ml}$. of deionized water. The solution was purified by precipitation of bismuth on the addition of $1.2 \mathrm{~g}$ of $1 \% \mathrm{~L}$ a in nitrate hexahydrate form (Aldrich, Milwaukee, WI, USA). Deionized water was obtained from a Mill-Q Plus water purifier (Millipore, Bedford, MA, USA). High-purity $\mathrm{HNO}_{3}$ was prepared in this laboratory by subboiling distillation of electronic grade $\mathrm{HNO}_{\text {s }}$ purchased from Dongwoo FineChem (Iksan, Korea).

\section{Results and Discussion}

Optimization of Hydride Generation. Various parameters were optimized individually while others were kept at fixed values. The optimized parameters included nitric acid concentration, sodium borohydride concentration, carrier argon flow rate and length of the mixing tube. Figure 2 shows the effect of nitric acid on the $0.1 \mathrm{ppb}$ Bi signal over the concentration range of $01.5 \mathrm{M}$. Only nitric acid was examined because the sample solution was prepared in 0.75 $M$ nitric acid. It can be observed from Figure 2 that the bismuthine generation efficiency is not significantly affected by the nitric acid concentration as long as the concentration is higher than $0.2 \mathrm{M} .0 .75 \mathrm{M}$ nitric acid was chosen so that

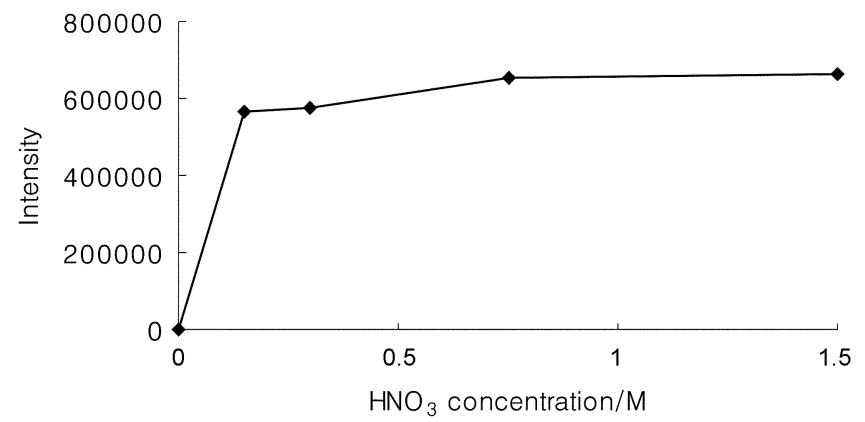

Figure 2. Fillect of $\mathrm{HNO}_{3}$ concentration on $0.1 \mathrm{ppb} \mathrm{Bi}$ signal (1\% $\mathrm{Naa3II} \mathrm{I}_{3}$.

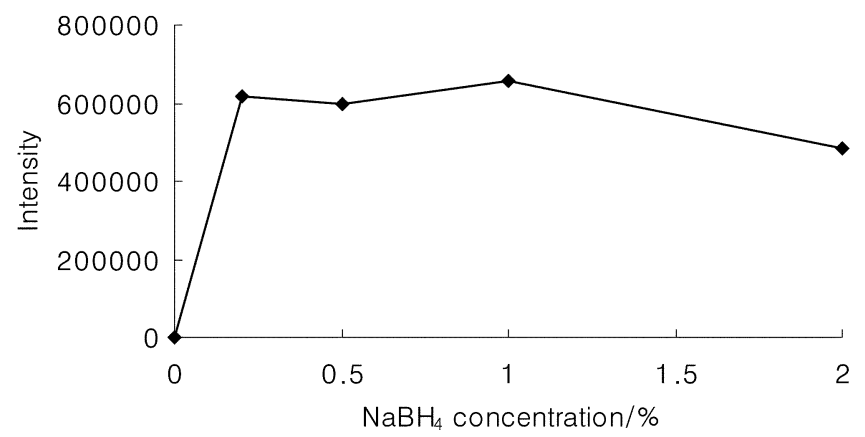

Figure 3. Efficet of $\mathrm{NaBH}_{+}$concentration on $0.1 \mathrm{ppb} \mathrm{Bi}$ signal $(0.75$ $\mathrm{MHNO}$ ). 


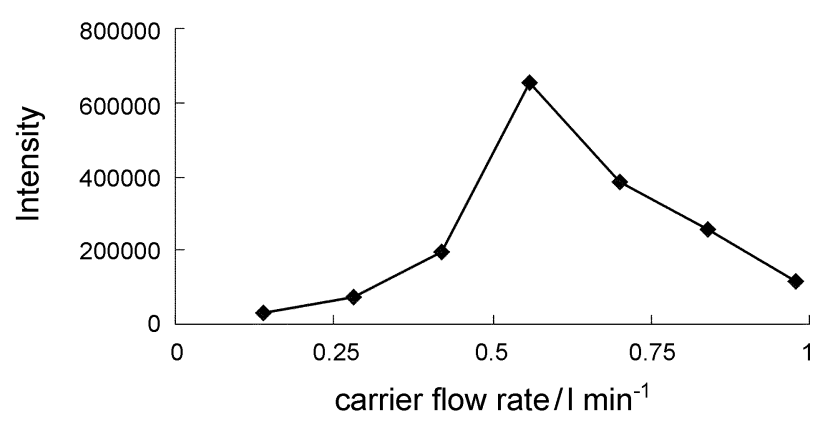

Figure 4. Etfect of carrice flow rate on $0.1 \mathrm{ppb}$ Bi signal ( $\mathrm{I}^{1 \%}$ $\mathrm{NaBH}_{+} 0.75 \mathrm{M} \mathrm{HNO}_{3}$ ).

no dilution of the sample solution would be necessary for the bydride generation. Figure 3 shows the effect of the sodium borohydride concentration on the Bi signal. It can be seen from Figure 3 that the Bi signal stays almost constant across the concentration interval between 0.2 and $1 \%$, and that it tends to drop at the higher concentration. Thus $1 \%$ sodium borohydride was chosen as an optimum concentration. Figure 4 shows the effect of the argon carrier flow rate on the Bi signal. ln common with the nebulization, the bismuth signal was very sensitive to the argon carrier flow rate. The optimum Ar carrier flow rate, for the hydride generation, was found to be $0.56[\mathrm{~L} / \mathrm{min}$, which was appreciably lower than the normal carrier flow rate of about $0.8 \mathrm{I} / \mathrm{m}$ in for the pneumatic nebulization. This difference can be explained as follows. The optimum carrier flow rate for the hydride generation is determined on the basis of an optimum plasma residence time with the minimum dilution of the hydride vapor, while that for the pneumatic nebulization is determined on the compromised condition for the efficient generation of aerosols and the optimum residence time in the plasma. length of the mixing tube determines the reaction time for which the hydride generation can take place. The Bi signal showed no significant difference as the mixing tube length was increased from $0.2 \mathrm{~m}$ to $2 \mathrm{~m}$. This means that the $\mathrm{Bi}$ hydride is formed rapidly and pretty stable in the gas phase. The length of the mixing tube was arbitrarily fixed to $0.5 \mathrm{~m}$ for the determination of $\mathrm{Bi}$ in the $\mathrm{Pb}$ solutions by the hydride generation.

Spectral Interference. It was thought at first that $\mathrm{Bi}$ could be determined without any interference effects by the nebulization of $10 \mu \mathrm{g} / \mathrm{mL} \mathrm{l}^{\mathrm{b}} \mathrm{b}$ solution ( 100 fold dilution of the $\mathrm{lb}$ solution for analysis). However, the $10 \mu \mathrm{g} / \mathrm{mL} \mathrm{Pb}$ matrix in the solution induced a significant amount of $\mathrm{PbH}^{+}$ molecular ion which gave rise to the spectral overlap with the ${ }^{20 / 9} \mathrm{Bi}$ analyte with an apparent concentration of $0.14 \mathrm{ng}$ $\mathrm{mL}$. Since $\mathrm{Bi}$ is one of the elements that can be reduced to

Table 2. Comparison ol hydride generation elficiency of Bi analyte with that of $\mathrm{Pb}$ matrix

\begin{tabular}{cccc}
\hline Solution & $\begin{array}{c}\text { Isotope } \\
\text { (abundance) }\end{array}$ & $\begin{array}{c}\text { Intinsity } \\
\text { (counts } / \mathrm{s})\end{array}$ & $\begin{array}{c}\text { Unit ppb intensity } \\
\text { (counts/s) }\end{array}$ \\
\hline $0.1 \mathrm{ng} / \mathrm{mL}, \mathrm{l3i}$ & $209(100 \%)$ & 680.000 & 6.800 .000 \\
\hline $100 \mathrm{Hg} / \mathrm{ml}, \mathrm{Pb}$ & $208(52.4 \%)$ & 3.900 .0000 & 74.4 \\
\hline
\end{tabular}

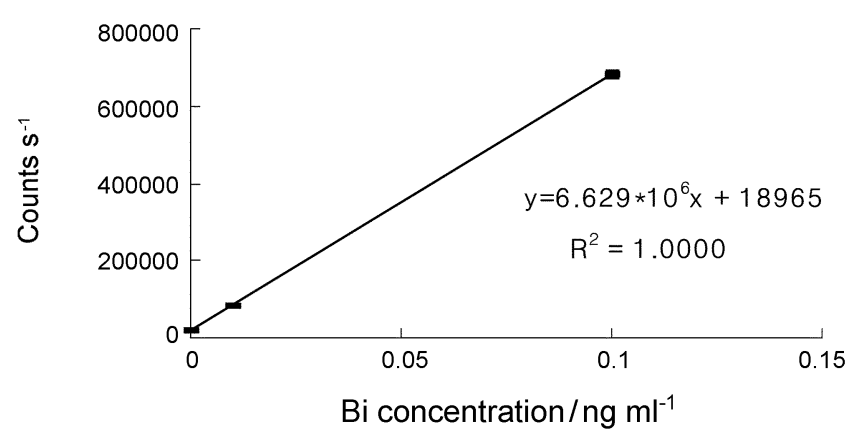

Figure 5. Standard calibration curve of Bi for sample introduction by hydride generation.

their corresponding hydrides, the hydride generation method was taken advantage of to separate the $\mathrm{Pb}$ matrix from the $\mathrm{Bi}$ analyte. The $\mathrm{Pb}$ matrix can be also reduced to $\mathrm{PbH}_{4}$ with the addition of an oxidizer for the oxidation of $\mathrm{Pb}$ in solution into the metastable $\mathrm{Pb}$ (IV) before reaction with $\mathrm{NaBH}_{4}$. Since no oxidizer was used for the Bi hydride generation in this work, the hydride generation efficiency of the $\mathrm{Pb}$ matrix was much lower than that of the Bi analyte as shown in Table 2. Using the hydride generation method, $0.1 \mathrm{ng} / \mathrm{mL}, \mathrm{Bi}$ standard solution produced the $\mathrm{Bi}$ signal of $0.68 \times 10^{6}$ counts/s (cps), while $100 \mu \mathrm{g} / \mathrm{ml}$. Pb solution produced ${ }^{208} \mathrm{~Pb}$ signal of $3.9 \times 10^{6} \mathrm{cps}$. Considering that $1 \mathrm{ng} / \mathrm{ml}, \mathrm{Pb}$ standard solution, by the nebulization, generates the ${ }^{208} \mathrm{~Pb}$ signal of $0.4 \times 10^{6} \mathrm{cps}$, the hydride generation of $100 \mu \mathrm{g} / \mathrm{mL}$. $\mathrm{Pb}$ solution introduces approximately the same amount of $\mathrm{Pb}$ into the plasma as the nebulization of $10 \mathrm{ng} / \mathrm{ml}$. Pb solution does. From the result that the nebulization of $10 \mu \mathrm{g} / \mathrm{ml}$. Pb solution induced the $\mathrm{PbH}^{-}$interferents corresponding to an apparent $\mathrm{Bi}$ concentration of $0.14 \mathrm{ng} / \mathrm{mL}$, it can be estimated that the hydride generation of $100 \mu \mathrm{g} / \mathrm{ml}$. Pb solution will produce the isobaric interference with an apparent $\mathrm{Bi}$ concentration of about $0.14 \mathrm{pg} / \mathrm{mL}$.

Analytical Results and Detection Limits. Figure 5 shows a standard calibration curve of $\mathrm{B} i$ for sample introduction by the hydride generation. 'The hydride generation was a highly efficient sample introduction method that gave about 17 times higher sensitivity than the nebulization. Jhough the spectral interference effect from the $\mathrm{l}^{\mathrm{l}} \mathrm{b}$ matrix by the hydride generation was found negligibly small, the $\mathrm{lb}$ matrix in the sample solution could cause a chemical interference in the $\mathrm{Bi}$ hydride generation. In order to negate the potential chemical interference, the standard addition method was used for the quantification. The standard addition method involves preparing a set of solutions in the sample matrix by adding known amounts of the standard to aliquots of the sample solution. Five sets of separately spiked sample solutions were prepared for the standard addition analysis of the primary $\mathrm{Pb}$ standard solution. Figure 6 and rigure 7 show the standard addition curves for the $\mathrm{Pb}$ solution at the concentrations of $100 \mu \mathrm{g} / \mathrm{mL}$ and $1000 \mu \mathrm{g} / \mathrm{mL}$. respectively. The two curves show different slopes indicating different degrees of the chemical interference from the two concentrations of the $\mathrm{Pb}$ matrix. Comparing slopes of the standard 


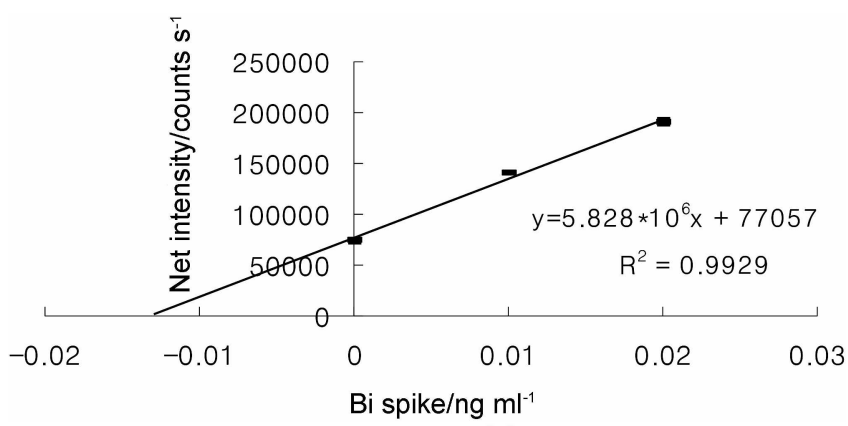

Figure 6. Standard addition curve for detemination of $\mathrm{Bi}$ in primary slandard solution $(100 \mu \mathrm{g} / \mathrm{ml}$. Pb).

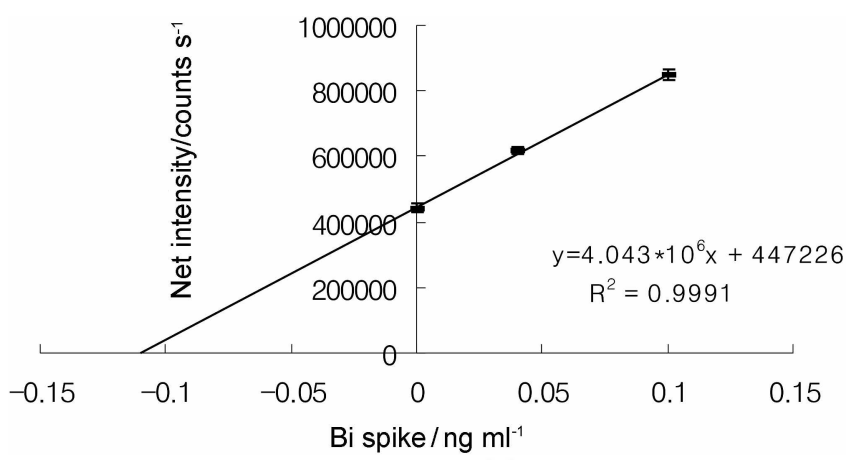

Figure 7. Standard addition curve for determination of $\mathrm{Bi}$ in primary standard solution (1000 $\mathrm{\mu g} / \mathrm{ml}, \mathrm{Pb})$.

addition curves in Figure 6 and Figure 7 with that of the standard calibration curve in Figure 5, it was estimated that the $\mathrm{Pb}$ matrix in the sample solution caused reduction of the Bi hydride generation efficiency by about $12 \%$ and $40 \%$ for the matrix concentration of 100 and $1000 \mu \mathrm{g} / \mathrm{mL}$, respectively. For comparison, a commercial $\mathrm{Pb}$ stock standard solution was analyzed for the Bi impurity by the standard addition method. As expected, the commercial standard solution contained higher $\mathrm{Bi}$ impurity than the prinary standard solution prepared in this laboratory (KRISS, Korea Research Institute of Standards and Science). Table 3 shows the determined concentrations of the Bi impurity in the 1000 $\mu \mathrm{g} / \mathrm{mL} \mathrm{Pb}$ solutions. The detection limit was determined by calculating the analyte concentration that yielded three times the standard deviation of the blank signal. The detection limit was calculated to be $0.3 \mathrm{pg} / \mathrm{mL}$ for sample solutions
Table 3. Analytical results for $1000 \mu \mathrm{g} / \mathrm{mL} \mathrm{Pb}$ standard solutions

\begin{tabular}{cc}
\hline Solutions & $\begin{array}{c}\text { I)etermined concentration } \\
\text { of } \mathrm{Bi} \text { impurity ( } \mathrm{ng} / \mathrm{mL} \text { ) }\end{array}$ \\
\hline KRISS Primary standard solution & $0.11 \pm 0.02$ \\
\hline Commercial standard solution & $1.6 \pm 0.2$ \\
\hline
\end{tabular}

without any matrix elements and $0.5 \mathrm{pg} / \mathrm{ml}$. for the sample solutions containing $1000 \mu \mathrm{g} / \mathrm{ml}, \mathrm{Pb}$ matrix.

\section{Conclusion}

Sub-ppb level of $\mathrm{Bi}$ impurity was determined in the $\mathrm{Pb}$ primary standard solution by ICP-MS with the sample introduction by the hydride generation, which effectively separated the $\mathrm{Bi}$ analyte from the $\mathrm{Pb}$ matrix and eliminated the spectral interference from ${ }^{2 \omega 8} \mathrm{PbH}^{\prime}$. The $\mathrm{Pb}$ matrix in the sample solution reduced the Bi hydride generation efficiency by about $12 \%$ for the matrix concentration of $100 \mu \mathrm{g} / \mathrm{mL}$ and about $40 \%$ for $1000 \mu \mathrm{g} / \mathrm{mL}$. The direct analysis of the $1000 \mu \mathrm{g} / \mathrm{mL} \mathrm{Pb}$ solutions with the hydride generation. together with its higher sample transport efficiency, provided much lower detection limit than that with the nebulization. The excellent detection limit of $0.5 \mathrm{pg} / \mathrm{mL}$ indicates that the present method could be applied to the determination of low $\mathrm{ng} / \mathrm{g}$ levels of $\mathrm{Bi}$ in high-purity $\mathrm{Pb}$ metals.

\section{References}

1. Watlers. R. L. The First Draft on the Isotope Dilution Mfass Spectrometry Protocol for /CP-MS: Vational Institule of Standards and Technology: Gaithersburg. MD. LISA. April 1995.

2. Sharp B. 1., J. Anct. At Spectrom. 1988. 3.613.

3. Douglas. D. 1.: Kerr. I. J. Anal At. Siectrom 1988. 3.749.

4. Beauchemin. 10.: McLaren. J. W.: Berman. S. S. Spectrochimica teta $1987 .+2 B .467$.

5. Richner. L'. J. Anal. dt. Spectom. 1993.8.927

6. Park. C. J. Bull. Koresn (When. Soc. 2002. 23.1541.

7. Vanhoe. II.: Goosens J.: Mons 1... Dame. R. J. Aned At. Spectrom. 1994.9 .177$.

8. Zhou. N.: lirech. W.: l.undberg. I: Anal. Chim. Acta 1983. I53. 23.

9. Cadore. S.: Baccan. N. J inat. At Spectrom. 1997. 12. 637.

10. Hall. G. E. M.: Pelchat. J. C. J. Anat. At. Spectrom. 1997. 12.97,

11. Powell. M. J.: Boomer. D. W. VicVicars R. J. And. Chem. 1986. 58.2864. 